\title{
Performance improvement of dye sensitized solar cell by using recycle material for counter electrode
}

\begin{abstract}
This project is to investigate the counter electrode material from two different carbon sources to fabricate dye sensitized solar cell (DSSC). The carbon sources are extracted from battery and pencil lead. The method to prepare the DSSC is through the conventional Dr.Blading method. The same method is also used for the counter electrode which uses carbon from recycle batteries, the carbon from pencil lead are scribbled onto the ITO glass to get a uniform coating. Both thickness of the counter electrode vary accordingly. The solar cells are then placed under outdoor solar irradiation and the output is taken every 10 minutes. Based on observation, the solar cells which have the carbon from batteries shows higher cell efficiency which is $8.2 \%$ with lower FF of 0.78 , compared to by using the pencil lead, the cell efficiency is only $7.23 \%$ but with a higher FF of 0.93 .
\end{abstract}

Keyword: Carbon; Dye sensitized solar cell (DSSC); Open circuit voltage; Solar cells efficiency 